\title{
Percutaneous Coronary Intervention (PCI) in the Era of Drug-Eluting Stents
}

\author{
NAM MOMENUZAMAN ${ }^{\mathrm{a}}$, F BEGUM $^{\mathrm{b}}$, F MALIK $^{\mathrm{c}}, \mathrm{S}_{\text {GHAFUR }}^{\mathrm{d}}$, N AHMED $^{\mathrm{b}}$, M BADIUZZAMAN $^{\mathrm{b}}$ \\ SM HOSSAIN $^{\mathrm{b}}$, MH RAHMAN ${ }^{\mathrm{d}}$, MIR NESARUDDIN AHMED $^{\mathrm{d}}$
}

\begin{abstract}
Summary:
Angioplasty opened the stenotic coronary artery but faced complication like abrupt vessel closure. Deployment of bare metal stent within the lesion solved the problem but another complication like restenosis emerged. Neointimal hyperplasia and vessel wall remodeling were identified as the underlying mechanism and lead to the development of drug eluting stent. In the selected patient group, drug eluting stent (sirolimus and paclitaxel) are proved to be extremely promising in respect to reduction of target vessel failure,
\end{abstract}

\section{Introduction}

Stent represents a major advance in the treatment of obstructive coronary artery disease since the advent of percutaneous transluminal coronary angioplasty $(\text { PTCA })^{1}$. PTCA opened the stenotic lesion in

a. Dr. N A M Momenuzzaman, D-Card, MD (Cardiology). Associate Professor and Chief of Cardiac Cath-Lab and Interventional Cardiology, Department of Cardiology, National Heart Foundation Hospital and Research Institute, Dhaka, Bangladesh

b. Dr. Fatema Begum, FCPS (Med), MD (Cardiology). Dr. Nazir Ahmed, MD (Cardiology), Dr. Md. Badiuzzaman, FCPS (Med), MD (Cardiology), Dr. S M Hossain, FCPS (Med), MD (Cardiology) Consultant Cardiologist, Department of Cardiology, National Heart Foundation Hospital and Research Institute, Dhaka, Bangladesh

c. Dr. Fazilatunnesa Malik, FCPS (Med), MRCP, FRCP. Associate Professor, Department of Cardiology, National Heart Foundation Hospital and Research Institute, Dhaka, Bangladesh

d. Dr. Shakil Ghafur, DTCD, MD (Cardiology), Dr Mir Nesaruddin Ahmed, MD (Cardiology) Assistant Professor and Dr. M H Rahman, FCPS (Med), Junior Consultatnt. Trainee in Invasive Cardiology, Department of Cardiology, National Heart Foundation Hospital and Research Institute, Dhaka, Bangladesh.

Address of correspondence: Dr. N A M Momenuzzaman, Associate Professor, Chief of Cardiac Cath-Lab and Interventional Cardiology, National Heart Foundation Hospital and Research Institute, Plot \# 7/2, Mirpur, Dhaka-1216, Phone 8053935-6, Ext:253, Fax: 880-2-8016694, Mobile: 01711529539, E-mail: momenuzzaman@hotmail.com

Received: 15 October, 2006

Accepted: 23 January, 2007 clinical restenosis (target vessel revascularization), binary restenosis, stent thrombosis and other parameters. Importantly initial positive outcome persisted in the subsequent later follow ups. Outcome in the unfavourable groups are also encouraging but needs few more studies and follow ups. There are scopes for future improvement also. With the immense success, drug eluting stents are increasingly used worldwide and becoming an integral part of percutaneous coronary intervention.

(J Bangladesh Coll Phys Surg 2007; 25 : 86-91)

coronary artery with potential promise but noted abrupt artery closure in $30 \%$ cases needing repeat procedure or emergent life saving bypass graft surgery. Advent of bare metal stent (BMS), an intravascular scaffold, mounted on a balloon and opened once inside the artery, virtually solved the problem by preventing vessel shrinkage ${ }^{2}$, but faced thrombotic complications (subacute stent thrombosis) in $20-25 \%$ cases $^{3}$ in the early part. High pressure stent deployment and use of antiplatelets, brought down the complications to ? $0.5 \% 1$.

Although the success and safety of stenting dramatically increased, in-stent restenosis persisted as a hindrance to stenting. Incidence varied from $8-80 \%$ at 06 months $^{2}$. Restenosis is higher in short lesions and large vessels and even more in high risk groups.

Restenosis is the arterial wall's healing response to mechanical (barotrauma) injury induced by PTCA or stent deployment, not a recurrence of coronary artery disease. Restenosis comprises two main processes, neointimal hyperplasia (arterial injury, immune response, smooth muscle cell migration/proliferation, intimal proliferation, extra-cellular matrix deposition, ECM and healing) and vessel remodeling (change in vessel dimension due to mechanical recoil from balloon expansion, less important in case of stenting) ${ }^{4}$.

Different treatment strategies were tried to reduce the restenosis rate with repeat balloon angioplasty, rotational atherectomy, laser angioplasty, cutting 
balloon angioplasty or repeat stenting and currently brachytherapy, but the rate still remains high. Mechanical approaches appear to be too simplistic to prevent in-stent restenosis as BMS induces neointimal hyperplasia though having positive scaffolding advantages. Interfering with molecular basis of this enhanced proliferative response appears to be a much more effective approach for modifying healing process after stenting ${ }^{2}$. Potential toxicity of systemic pharmacotherapy and inadequate drug concentration at the injury site lead local drug delivery system to emerge ${ }^{1}$. Although catheter-based drug delivery has mixed results (rapid washout of the drug and the potential flow or pressure mediated vessel wall injury $)^{5}$, recently the emergence of drug-eluting stent (DES) technology offered a new perspective for the pharmacological prevention of restenosis ${ }^{1}$.

The application of DES technology to improve clinical outcomes following percutaneous coronary intervention (PCI) represents one of the greatest success stories in cardiology. A variety of drugs were tested which interrupt the biological process that caused restenosis. DES is a normal metal stent coated with such pharmacological agent and proved to be extremely successful in reducing restenosis from the $20-30 \%$ range to a single digit 6,7 . There are three major component of DES; type of stents that carries the drug coating, method by which the drug is delivered to the arterial wall for time release (polymer or other) and the drug itself (to prevent restenosis) ${ }^{1}$.

Although research and clinical practice employing DES started few years back, FDA approved first DES to use in USA in April 2003 and the practice is ever increasing every year all over the world ${ }^{8}$. DES has been classified according to its properties; antiinfammatory (corticosteroids, tranilast), antithrombotic (NO, GP IIb/IIIa inhibitors, hirudin, iloprost), immunosuppressive (sirolimus, rapamycin analogue evorlimus, ABT-578, tacrolimus, mycophenolic acid), anti-proliferative (paclitaxel, angiopeptin), modulator of ECM (batimastat) and promoter of healing (NO, VEGF, estradiol) ${ }^{1,9}$. Their action is harmless to normal cells.

Among them, two DES had undergone a number of studies and gained acceptance and increasingly practiced, eg. sirolimus eluting stents (SES) and paclitaxel eluting stents (PES) ${ }^{9}$. Few more like ABT 578, evorlimus and trapidil eluting stents have entered the market and their use is increasing.

\section{Sirolimus}

Sirolimus (Rapamycin) is a macrolide antibiotic, initially used for prophylaxis against renal transplant rejection ${ }^{2}$. Shortly afterwards, the SES was implanted in human coronary arteries ${ }^{10}$.

Mechanism of action ${ }^{2}$ :

Rapamycin is actually a pro-drug that ultimately binds to a specific cell cycle-regulatory protein, the mTOR and inhibits its activation. mTOR is involved in the transition between the $G_{1}$ and $S$ phase where DNA replication occurs and lead to cell division. Rapamycin, thus have a cytostatic effect and induce cell cycle arrest in late $G_{1}$ phase and may inhibit cellular migration at higher doses and thus inhibits all phases of restenosis cascade.

Rapamycin acts at very low nanomolar level, 140 $? \mathrm{gm} / \mathrm{cm}^{2}(180 ? \mathrm{gm} / 18 \mathrm{~mm} \text { stents })^{11}$. Both fast-release (< 15-day) and slow-release (? 28-day) formulations are available. Only slow release formulations were randomly tested and now commercially available.

Several clinical studies were conducted employing SES. First of such study was FIM (First-In-Man) in 1999 and another study RAVEL (RAndomized study with the sirolimus-eluting Bx VELocity balloonexpandable stent).

Recently published one year outcome of SIRIUS (SIRolImUS-coated Bx Velocity stent in the treatment of patients with de novo coronary artery lesions) trial shows immense benefit of SES over BMS $^{6}$. This multicenter randomized, double-blind study enrolled patients with more liberal inclusion criteria and dividing patients randomly undergoing PCI assigned to SES and control BMS. The primary end points were target vessel failure, TVF (includes TVR, cardiac death, Q-wave and non-Q-wave MI not clearly attributed to a vessel other than the target vessel) and clinical restenosis (target lesion revascularization, TLR). Secondary end points were MACE or repeat TLR at 30 days, 9 and 12 months after index procedure.

At 9 month follow-up clinical restenosis, defined by TLR was significantly lower in the SES group as well as TVF and stent thrombosis rates. In-stent binary restenosis rate and in-segment restenosis were also markedly lowered ${ }^{12}$. At 12 months, this significant difference remained with only a minimal increase in the end points in either group. Strikingly TLR rises but still lower than control group (Table I). 


\section{Table-I}

Comparison of key outcomes in SIRIUS Trial

\begin{tabular}{lccc} 
& SES & BMS & Significance (p) \\
\hline TLR at 9 month & $4.10 \%$ & $16.60 \%$ & $<0.001$ \\
TVF at 9 month & $8.80 \%$ & $21.00 \%$ & $<0.001$ \\
Stent thrombosis at 9 month & $0.40 \%$ & $0.80 \%$ & $\mathrm{NS}$ \\
In-stent binary restenosis at 9 month & $3.20 \%$ & $35.40 \%$ & $\mathrm{NS}$ \\
In-segment restenosis at 9 month & $8.90 \%$ & $36.30 \%$ & $\mathrm{NS}$ \\
Rise of TLR at 12 month & $4.1 \%$ to $4.9 \%$ & $16.6 \%$ to $20.0 \%$ & \\
\hline
\end{tabular}

SES: Sirulimus eluting stent, BMS: Bare metal stent, TLR: Target lesion revascularization, TVF: Target vessel failure

In-stent late loss was $0.17 \mathrm{~mm}$ and in-lesion late loss was $0.25 \mathrm{~mm}$. DM, reference-vessel diameter and lesion length were the significant determinant of TLR. $70-80 \%$ relative reduction in clinical restenosis was achieved with SES irrespective of their presence. The restenosis rate was a bit higher in SIRIUS trial to that of RAVEL because of inclusion of more adverse baseline characteristics for restenosis.

The SIRIUS trial confirms benefit with SES in a group of patients and lesions at increased risk of restenosis for up to 1 year with out any evidence of untoward effect. Similar improvement in outcome in other patient groups at even higher risk for restenosis, including in-stent restenosis, total occlusions and multivessel stenting remains to be determined.

\section{Paclitaxel}

Paclitaxel are effectively used in the treatment of various cancers. This is a deterpenoid compound with a nucleus and a side chain. Modification of the side chain produces a more potent analogue, docetaxol. Both have unique pharmacological action as inhibitors of mitosis by promoting microtubule formation and assembly and antagonizing disassembly, which halts mitosis $\left(\mathrm{G}_{2} / \mathrm{M} \text { phase }\right)^{2}$. As such they lead to reduced vascular cell proliferation, migration and signal transduction ${ }^{13}$.

A series of clinical trial (TAXUS-I through IV) were designed to test the feasibility and effectiveness of polymer based PES with various release kinetics comparing with BMS in a variety of clinical settings.

\section{TAXUS-IV ${ }^{7}$}

This is a pivotal large, double blind, multicenter, randomized trial testing the efficiency of slow release
(SR) PES in patients with single de novo lesion by comparing control BMS. With some major exclusion criteria, randomization was done according to DM, vessel size and type of stents. Clinical follow up were scheduled at 1, 3, 4 and 9 months and yearly thereafter for 5 years. Angiographic follow-up were done at 9 month and 12 month. The primary end point was ischemia driven TVR at 9 months.

Follow up at 12 month revealed the significant reduction of TLR, TVR and restenosis frequency in PES and as such the rates of composite TVF and MACE. The rates of cardiac death, MI and stent thrombosis (all occurred within first 6 months, no late stent thrombosis occurred 6 months after clopidogrel discontinuation) were similar between two groups (Table II).

\section{Table II}

Comparison of key outcomes in TAXUS IV Trial

\begin{tabular}{lccc} 
& PES & BMS & Significance (p) \\
\hline TLR reduction at 12 month & $4.40 \%$ & $15.10 \%$ & $<0.001$ \\
TVR reduction at 12 month & $7.10 \%$ & $17.10 \%$ & $<0.001$ \\
Restenosis at 12 month & $7.60 \%$ & $26.60 \%$ & $<0.001$ \\
\hline
\end{tabular}

PES: Paclitaxel eluting stent, BMS: Bare metal stent, TLR: Target lesion revascularization, TVR: Target vessel revascularization

The relative reduction in TLR rates at 12 month with PES was independent of vessel location, reference vessel diameter, lesion length, diabetic status, female gender and no prior myocardial infarction. There was no safety concern emerged in the PES group during this extended follow up period. The PES (TAXUS Stent) markedly reduced the need for repeat 
revascularization procedure particularly if adjunctive pharmacological strategies can curtail periprocedural adverse events.

Absolute and relative rate of composite MACE increased in PES group observed during follow up period. So extended follow up for several years and enrollment of greater number of patients are needed to study any late hazards. Additional studies are also needed to demonstrate the safety and efficacy of this device in more complex and high-risk lesions not included in this study.

\section{Drug-Eluting Stents: what future holds}

Initial randomized trials employing DES, enrolled patients with single de novo lesions, intermediate length, larger vessel diameter, single stent in the absence of high risk criterias $6,7,8$. Restenosis rate in current trials seems to be higher, than previous because of enrolling patients with more risk factors, but still significantly less when compared with $\mathrm{BMS}^{6}$.

Although $70-80 \%$ relative reduction in clinical restenosis with DES irrespective of lesion length, reference vessel diameter, diabetes, LAD lesion, post stent restenosis (few examples of high risk lesions), we can not recommend routine use of DES in such patient subgroups ${ }^{6,12}$. We need to continue the observational follow up for couple of years more whether the same persists. Again it is time to determine definitively whether similar marked improvement with DES will be observed in other groups at even higher risk for restenosis, including those with in-stent restenosis, total occlusion, multi lesion/multi vessel disease (specially in diabetics), large $(>3.5 \mathrm{~mm})$ and small $(<2.5 \mathrm{~mm})$ vessel diameter, saphenous vein grafts, diffuse disease (full metal jackets), longer lesions, left main disease, side branch or bifurcating lesions, post brachytherapy restenosis and also in acute MI (primary PTCA) $)^{6,8,12}$.

\section{Concerns and controversies}

Till today, there are few concerns about the immensely successful DES, like safety profile of stents coated with potent antimitotic agents, polymer related hazards, accelerated atherosclerosis, delayed stent thrombosis, lack of a long-term effect on restenosis ( $>2$ years) and being very expensive. There are few limitations as well like they can not be used in patients with allergy to sirolimus, polymethacrylates or polyelefins, requires anti platelet medication for several months (particularly for paclitaxel), potential adverse effects of sirolimus, procedural less versatility, originally more expensive.

\section{Future development}

Although the DES has gained acceptance for use in the treatment of obstructive coronary lesion, continuous endeavor is going on to improve this technology further to improves its restenosis coverage. Following are the proposals:

Pharmacological modulation: Stent coated with Estradiol improves vascular healing, reduce SMC migration and proliferation and promote local angiogenesis ${ }^{14}$.

Tissue engineering: Tissue engineered perivascular endothelial cell implants can identify heparin sulphate proteoglycan perlecan as a potent inhibitor of neointimal hyperplasia after deep vascular injury ${ }^{15}$.

Gene and stem cell therapies: Endothelial-derived NO helps in vascular healing by attenuating inflammation and inhibiting SMC proliferation and migration ${ }^{16}$. Genetic intervention induces increased vascular reactivity of the injured vessel resulting in reduced neointimal hyperplasia.

Vascular endothelial growth factor (VEGF) is a potent vascular permeability factor and is an endogenous regulator of endothelial integrity after injury and thus protect artery from disease progression ${ }^{17}$. Animal studies revealed positive outcome after balloon and stent injury following VEGF-gene-eluting stents with a future prospective of human trial ${ }^{18,19,20,21}$.

The transplantation of endothelial progenitor cells (EPCs) can enhance endothelial cell regeneration, neovascularization or both ${ }^{2}$.

Somatic stem cells in the bone marrow are capable of differentiating into vascular endothelial cells and SMCs, and home to sites of vascular damage following vascular damage. Antibodies against their membrane receptors at the site of vascular injury has been proposed ${ }^{2}$. Antibody-coated stents implanted in human coronaries in multicenter pilot study (HEALING) without adverse reactions, but long-term outcomes are pending. 
Procedural modification: Direct stenting is associated with more than $80 \%$ reduction in the vessel wall regeneration with a two fold reduction in intimal thickening as compared with predilatation ${ }^{22}$. Improvement of stent and balloon design and deployment technique is going on to reduce their biological repair response.

Combination chemotherapy: This is effective in reducing neointimal thickening. Combination of hirudin and ilioprost blended with polylactic acid polymer can be loaded onto a stent ${ }^{2}$. Paclitaxel-NO donor conjugate-eluting stent is more beneficent than PES alone ${ }^{2}$.

Gene therapy combined with pharmacologic therapy modulate distinct ligand-receptor signaling system. Oral Imatinib mesylate (STI571/gleevec) improves the efficacy of local intravascular VEGF-C gene transfer in reducing neointimal growth ${ }^{23}$.

Customized stents: These stents allow both temporal and spatial control of drug release. They cause early release of antithrombotic prohealing agent in the luminal surface and thus promote healing and stent endothelialization, where as an anti proliferative agent (cell cycle inhibitors) could be released intramurally and slowly ${ }^{2}$. The Conor stents has individual polymer inlays for drug reservoirs that can be loaded with different compounds ${ }^{24}$.

Polymeric biodegradable stents: Noninflammatory biodegradable polymeric stent, where polymer dissolves slowly after implantation are promising technologies that can be loaded with large amount of drugs or multiple agents ${ }^{2,8}$. These stent provide initial scaffolding support to prevent vessel recoil and negative recoil without undesirable continuous vessel trauma caused by a permanent foreign body and adverse effect of retained drug or polymer.

Improvement of stent: Numerous new platforms, such as ultra-low-profile, thin-strut stent built from cobalt chromium and other novel materials, may reduce vascular injury and improve deliverability ${ }^{8}$.

\section{Conclusion}

Drug eluting stent promises to be a huge leap in the treatment of obstructive coronary atherosclerotic disease. DES is an emerging technology that has wide scale of implications with scope for future development. Evidence on the clinical efficacy and safety of SES and PES are continuously being released. Positive outcome are seen even in the unfavorable candidates. Given the trend of cardiovascular disease in the western world, DES usages will continue to grow.

\section{Reference:}

1. Sousa JE, Serruys PW, Costa MA. New Fronitiers in Cardiology. Drug-Eluting Stents: Part I. Circulation 2003; 107: 2274-2279

2. Costa MA, Simon DI: Molecular basis of restenosis and Drug-Eluting Stents. Circulation 2005; 111: 2257-2273

3. Serruys PW, Strauss BH, van Beusekom, et al. Stenting of coronary arteries: has a modern Pandora's box has been opened? J Am Coll Cardiol 1991; 17: 143B-154B

4. Sousa JE, Costa MA, Tuzuc EM, et al. New frontier in interventional cardiology. Circulation 2005; 111: 671-681

5. Lincoff AM, Topol EJ, Ellis SG. Local drug delivery for the prevention of restenosis: fact, fancy, and future. Circulation 1994; 90: 2070-2084

6. Holmes DR, Leon MB, Moses JW, et al. Analysis of 1-Year clinical outcome in the SIRIUS trial: A randomized trial of a Sirolimus-Eluting Stent versus a Standard Stent in patients at High Risk for Coronary Restenosis. Circulation 2004; 109: 634-640

7. Stone GW, Ellis SG, Cox DA, et al. One-Year Results With the Slow-Release, Polymer-Based, Paclitaxel-Eluting TAXUS Stent: The TAXUS-IV Trial. Circulation 2004; 109: 1942-1947

8. Teirstein PS. A Chicken in Every Pot and a Drug-Eluting Stent in Every Lesion. Circulation 2004; 109: 1906-1918

9. Sousa JE, Serruys PW, Costa DA, et al. New Frontiers in Cardiology. Drug-Eluting Stents: Part II. Circulation 2003; 107: 2383-2389

10. Sousa JE, Costa MA, Abizaid A. et al. Lack of neointimal proliferation after implantation of sirolimus-coated stents in human coronary arteries: a quantitative angiography and three-dimensional intravascular ultrasound study. Circulation 2001; 103: 192-195

11. Sujuki T, Kopia G, Hayashi S, et al. Stent based delivery of sirolimus reduces neointimal formation in a procine coronary model. Circulation 2001; 104: 1188-1193

12. Moses JW, Leon MB, Pompa JJ, et al. Sirolimus-eluting stents versus standard stents in patients with stenosis in a native coronary artery. N Eng J Med 2003; 349: 1315-1323

13. Sollott SJ, Cheng L, Pauly RR, et al. Taxol inhibits neointimal smooth muscle cell proliferation after angioplasty in the rat. J Clin Invest. 1995; 95: 1869-1876

14. Geralds P, Sirois MG, Bernatchez PN, et al. Esrogen regulation of endothelial and smooth muscle cell migration 
and proliferation: role of $\mathrm{p} 38$ and p42/44 mitogen-activated protein kinase. Arterioscler Thromb Vasc Biol 2002; 22: $1585-1590$

15. Nugent MA, Nugent HA, Iozzo RV, et al. Perlecan is required to inhibit thrombosis after deep vascular injury and contributes to endothelial cell mediated inhibition of intimal hyperplasia. Proc Natl Acad Sci USA 2000; 97: 6722-6727

16. von der Leyen H, Gibbons G, Morishita R, et al. Gene therapy inhibiting neointimal vascular lesion: in vivo transfer of endothelial cell nitric oxide synthase gene. Proc Natl Acad Sci USA 1995; 92: 1137-1141

17. Folkman J. Angiogenesis in cancer, vascular, rheumatoid and other disease. Nat Med 1995; 1: 27-31

18. Van Belle E, Tio FO, Chen D, et al. Passivation of metallic stents after arterial gene transfer of pH VEGF165 inhibits thrombus formation and intimal thickening. J Am Coll Cardiol 1997; 29: 1371-1379

19. Van Belle E, Maillard L, Tio FO, et al. Accelerated endothelializaiton by local delivery of recombinant human vascular endothelial growth factor reduces in-stent intimal formation. Biochem Biophys Res Commun 1997; 235: 311316
20. Asahara T, Bauters C, Pastore C, et al. Local delivery of vascular endothelial growth factor accelerates reendothelialization and attenuates intimal hyperplasia in balloon injured rat carotid artery. Circulation 1995; 91: 2793-2801

21. Walter DH, Cezna M, Diaz-Sandoval L, et al. Local gene transfer of ph VEGF-2 plasmid by gene-eluting stents: an alternative strategy for inhibition of restenosis. Circulation 2004; 110: 36-45

22. Rogers C, Parikh S, Seifert P, et al. Endogenous cell seeding: remnant endothelium after stenting enhances vascular repair. Circulation 1996; 94: 2909-2914

23. Lepanen O, Rutanen J, Hiltunen MO, et al. Oral imatinib mesylate (STI571/gleeve) improves the efficacy of local intravascular vascular endothelial growth factor-C gene transfer in reducing neointimal growth in hypercholesterolemic rabbits. Circulation.2004; 109: 1140146

24. Finkelstein A, McClean D, Kar S, et al. Local drug delivery via a coronary stent with programmable release pharmacokinetics. Circulation 2003; 107: 777-784 